\title{
Universidad y producción social del hábitat: Investigación, Formación y Acción
}

\section{University and social production of Habitat: research, training and action}

\author{
María Bernabela Pelli* y Esteban de Manuel Jerez**
}

Hábitat y Sociedad (ISSN 2173-125X), n. ${ }^{\circ}$ 10, noviembre de 2017, pp. 5-12. http://dx.doi.org/10.12795/HabitatySociedad.2017.i10.01

\footnotetext{
Universidad Nacional del Nordeste (Argentina).

** Universidad de Sevilla (España).
}

L a necesidad de construir nuevos paradigmas de comprensión e intervención en el hábitat, que aborden su complejidad, presenta un reto para la universidad. Es preciso construir conocimiento tejiendo áreas especializadas que aborden el hábitat desde lo físico, lo social, lo económico, lo simbólico, lo jurídico y lo político. El hábitat social está llamado a constituirse en un espacio transdisciplinario de conocimiento e intervención. Y en esta construcción la universidad está llamada a jugar un papel fundamental poniendo en juego las tres dimensiones de su función social: investigación, docencia y extensión universitaria. La investigación en la acción participativa y los modelos de aprendizaje-servicio permiten explorar caminos de construcción colectiva del conocimiento en contextos de intervención, con todos los agentes sociales implicados. La praxis se convierte en lugar privilegiado para el aprendizaje y la generación de conocimiento.

En América latina existe una rica y larga experiencia de extensión universitaria y de creación de espacios de aprendizaje que traen a la academia la problemática social. Son espacios formativos en los que las y los estudiantes toman contacto con la realidad del hábitat popular, normalmente autoproducido sin ningún tipo de apoyo técnico, muchas veces al margen de la ciudad planificada. Aprenden aportando conocimiento situado y descubren herramientas para interpretar e intervenir en esos contextos de escasos recursos, donde la intervención va más allá de la mejora física del entorno. Es habitual que sea en estos espacios de la periferia de la universidad donde esta descubra una oportunidad de desarrollar su función social. Pero también ese acercamiento puede venir desde la iniciativa de grupos de investigación que asumen el hábitat socialmente producido como objeto de estudio y que en la interacción con los y las habitantes empiezan a descubrir que el conocimiento necesario para interpretar la problemática de estos asentamientos desborda las disciplinas e incluso la academia, pues hay conocimientos populares, producto de la experiencia de habitar, que son imprescindibles.

Cuando la universidad no cuenta con espacios de extensión universitaria preparados para canalizar necesidades sociales vinculadas al hábitat y darles respuesta en forma de conocimiento transferible y apropiable, como ha sido el caso de España hasta muy recientemente, ha sido preciso crear ámbitos institucionales, asociaciones universitarias, por iniciativa de estudiantes y profesores, para dar respuesta tanto a la demanda social insatisfecha de vivienda y ciudad, como a la demanda de una par- 
te de la comunidad universitaria de contar con herramientas y conocimientos socialmente pertinentes para abordar la realidad. Ha sido ese el caso en la Escuela de Arquitectura de Sevilla del nacimiento de la ONGD universitaria Arquitectura y Compromiso Social (1993-2016). Pero también ha sido posible encontrar espacios, como en América latina, para plantear asignaturas optativas desde las que abordar la realidad de la problemática del hábitat socialmente producido como respuesta a la inaccesibilidad del mercado por una parte significativa de la población y la insuficiente respuesta por parte de los poderes públicos. Algunas de estas iniciativas han tenido una vida efímera y otras se han consolidado y han llegado a integrarse formalmente en el currículum, como asignaturas obligatorias u optativas. Junto con el diseño de materias específicas para abordar la problemática del hábitat social, también encontramos experiencias que han introducido estos temas como contenidos transversales de asignaturas obligatorias del currículum. La oferta de cursos de posgrado ha sido otra de las vías utilizadas por universitarios comprometidos para dar respuesta a las necesidades de formación. Unos han surgido con vocación disciplinar, dirigidos fundamentalmente a arquitectos, ingenieros, sociólogos, educadores sociales, ambientólogos, juristas o economistas. Y otros lo han hecho con decida vocación transdisciplinar.

Todas estas experiencias, minoritarias y contrahegemónicas, se ven reforzadas cuando se articulan en redes, como es el caso de la Red Universitaria Latinoamericana de Cátedras de Vivienda. En España podemos encontrar iniciativas paralelas que terminan por converger en el ámbito de la acción, de la investigación o de la intervención, provenientes de distintas disciplinas. En el campo de la sociología encontramos redes de investigación-acción participativa vinculadas al desarrollo local, que dan soporte epistemológico y metodológico a los profesionales del campo social para diseñar procesos participativos de intervención en el hábitat. En el campo de la arquitectura encontramos redes de acción como Arquitectura Social, surgidas del ámbito asociativo, profesionales como Arquitecturas Colectivas, y universitarias, como la que promueve los congresos Arcadia (Arquitectura y Cooperación al Desarrollo). Bajo el paraguas del aprendizaje-servicio, de la investigación-acción participativa, de la cooperación al desarrollo, de la formación para la producción y gestión social del hábitat, se articulan espacios de intercambio de experiencias y conocimientos, con ofertas de cursos de grado y posgrado que vinculan la generación del conocimiento a la reflexión en la acción participativa para la mejora del hábitat con criterios de sustentabilidad.

Este número surge con el propósito de visibilizar estas iniciativas y de contribuir a la construcción de las bases teórico-metodológicas que permiten dar respuesta a esta necesidad de interacción universidad-sociedad, para afrontar la transformación de nuestro hábitat social en clave de participación democrática, equidad social, activación de la economía local y equilibrio ambiental. En este sentido, hicimos una convocatoria a la presentación de artículos científicos que expusieran tanto planteamientos teóricos como resultados de procesos de investigación. La respuesta ha sido generosa y recoge aportaciones argentinas, mexicanas, chilenas, uruguayas, brasileñas y españolas.

Los dos artículos que abren el número desarrollan los pilares epistemológicos y metodológicos desde los que plantear tanto la intervención en procesos sociales de transformación del hábitat como la formación de los profesionales que los acompañen. Lo hacen desde la 
reflexión sobre la práctica, tras una dilatada experiencia de intervención formativa.

La Dra. en Geografía Elena Lucca, en "Estrategias y metodologías de formación de equipos interdisciplinarios para trabajar en hábitat”, aborda el tema desde la necesidad de crear espacios en la universidad desde los que ir construyendo una mirada común sobre el territorio a partir de la interacción de las miradas disciplinarias, superándolas para crear una mirada integrada, al tiempo que aporta unas pautas para intervenir en el territorio en diálogo con los habitantes. La Extensión Universitaria le ha posibilitado la creación de esos espacios de formación e intervención participativa. El análisis de los datos registrados durante más de quince años de experiencia, permiten a la autora extraer categorías conceptuales y metodológicas. Este artículo se complementa con la lectura que la Dra. en Ciencias Ambientales Laura Pérez Prieto realiza del libro de Elena Lucca La gestión de los territorios. La Cosecha escondida o percepción ambiental de los espacios. En el mismo, la autora desarrolla, desde el enfoque ecológico o de sostenibilidad fuerte, una metodología para trabajar de forma participada la construcción de Agendas Locales 21. Plantea la necesidad de religar el lazo roto entre sociedad y naturaleza a partir de la construcción con las y los habitantes de una nueva mirada sobre su territorio que les permita construir herramientas para la gestión sustentable de sus recursos.

Los doctores en sociología Manuel Montañés Serrano y Pedro Martín Gutiérrez presentan una construcción epistemológica y metodológica, extraída de la experiencia de impartición de cursos de posgrado. Cursos promovidos por el sociólogo Tomás Rodríguez Villasante, que en 1995-96 dirigió en la Universidad Autónoma de Madrid la primera edición del curso "Especialista Universitario en Investigación Participativa y Gestión Local", que evolucionaría a máster e iría adaptando su denominación y su metodología, desde la Investigación Acción Participativa (IAP) a la sociopraxis. Estos cursos han estado siempre vinculados a convenios con municipios que han propiciado escenarios reales de formación-acción tutorizada a los estudiantes. De este modo lograron crear una sinergia que ha permitido a los municipios tener un aporte de la universidad, útil a intervenciones en curso en su territorio, al tiempo que, en virtud de dichos convenios, se contribuía a la sostenibilidad económica de la oferta formativa universitaria y se propiciaba ese espacio o interfaz propicio al aprendizaje de metodologías participativas. Es interesante destacar que este planteamiento se ha replicado por discípulos de Villasante en otros territorios, multiplicando así su incidencia en multitud de procesos participativos locales. Asimismo, es importante destacar la capacidad de generar red entre investigadores y profesionales que ha tenido este curso.

Desde Argentina, ciudad de Córdoba, los arquitectos Aurelio Ferrero y Gustavo Rebord, en su artículo "Participación: asignatura pendiente de los arquitectos", proponen incursionar sobre otras posibilidades formativas de los arquitectos, frente a la realidad urbana actual y un perfil profesional adecuado para actuar en este escenario. En este sentido presentan la Red ULACAV (Red Universitaria Latinoamericana de Cátedras de Vivienda), que reúne diferentes cátedras de grado y posgrado, que orientan la formación hacia el trabajo en el campo del hábitat popular, en el que la participación es imprescindible. Se completa el artículo con la propia experiencia en el ámbito de la Universidad Nacional de Córdoba, a partir de un recorrido por cuatro prácticas docentes desarrolladas durante los últimos 26 años. 
Estos artículos se completan con el escrito del doctor arquitecto Víctor S. Pelli, "La pobreza, la universidad y el oficio de arquitecto en la América latina de comienzos del siglo XXI", sobre la base de la conferencia del mismo título pronunciada como ponencia invitada para la inauguración del congreso Arcadia IV, en A Coruña, 2016. Como los anteriores, es una sistematización de una práctica profesional dirigida a dar respuesta profesional, desde la universidad, al problema del acceso a la vivienda para esa gran parte de la población que carece de recursos suficientes para acceder a ella por sí misma de manera mínimamente satisfactoria. Partiendo de una caracterización de la demanda insatisfecha de la vivienda en el ámbito latinoamericano, en el que ha desarrollado su trayectoria el autor, plantea las bases desde las que la universidad puede dar una respuesta adecuada y sistematiza las distintas instancias promovidas en su ámbito para hacerlo, tanto desde la intervención directa, acompañando procesos participativos de mejora del hábitat, como desde la investigación y la docencia. Desde esa panorámica Víctor Pelli termina elaborando unas propuestas con criterios para abordar la gestión del acceso a la vivienda para los sectores de población empobrecidos. Termina su trayectoria profesional, por ahora, promoviendo con su equipo la Maestría en Gestión y Desarrollo de la Vivienda Social. En este marco se celebró un seminario internacional cuyos aportes quedaron plasmados en el libro Experiencias Habitacionales Significativas en Latinoamérica y España. Se presenta en esta revista, con una reseña realizada por la doctora arquitecta Venettia Romagnoli.

En desarrollo e historias de vidas paralelas, desde México, el doctor arquitecto Enrique Ortiz Flores, entre anécdotas y reflexiones sobre la realidad que le tocó vivir y en la que logró incidir, plasma las principales experiencias y los aprendizajes de su vida dedicada a luchar por el reconocimiento y la obtención de una vivienda digna para todos en el libro Hacia un hábitat para el Buen Vivir, Andanzas compartidas de un caracol peregrino, cuya reseña estuvo a cargo de la magíster en Derecho Maria Silvia Emanuelli.

A continuación, presentamos cinco experiencias referentes de formación, vinculadas a la extensión universitaria.

La magíster arquitecta Beatriz H. Pedro, en su artículo "Espacios académicos que aborden la formación de los profesionales que intervienen en el hábitat desde el compromiso con los intereses y necesidades populares", presenta la propuesta pedagógica del Taller Libre de Proyecto Social, que se desarrolla dentro de la Universidad de Buenos Aires y en el territorio del Área Metropolitana Buenos Aires. Plantea una forma de abordaje de las problemáticas sociales del hábitat en la formación de los arquitectos y diseñadores, a la que han denominado Enfoque social del diseño y el proyecto. La propuesta cuestiona los parámetros actuales de la formación y sus criterios de valoración y validación proyectual.

El doctor arquitecto Plácido Lizancos, junto con Luis Lage, Francisco Raigón, Vanessa Míguez y Alberto Varela, en "Formulación de una acción innovadora de aprendizaje-servicio enfocada a la habitabilidad básica. Una experiencia interuniversitaria en Maputo (Mozambique)", relatan la posibilidad que ofrece Kaya Clínica, como un sistema de aprendizaje y servicio universitario, implementado desde diciembre de 2015, por dos instituciones universitarias, la Universidade da Coruña (España) y la Universidade Eduardo Mondlane (Mozambique), para el ejercicio de la habitabilidad básica. Experimentan con estudiantes 
la intervención en un entorno social y físico verídico, como es el de la atención a las necesidades habitacionales de las personas en situación de pobreza, que pese a autogestionar su hábitat han sido incapaces de acceder a una asistencia técnica adecuada.

Los doctores arquitectos Manuel Martínez Hernández y Vicente J. Díaz García, en "Hábitat y desarrollo. Explorando caminos de innovación educativa en arquitectura y urbanismo", presentan el marco teórico y metodológico y analizan los resultados de la asignatura que, con este nombre, imparten en la ETS de Arquitectura de Las Palmas, en Canarias, desde el año 2001. La ubican dentro del panorama emergente en España, durante los últimos 20 años, de asignaturas de grado y de posgrado que abordan desde la arquitectura temáticas como habitabilidad básica, cooperación al desarrollo de asentamientos humanos, participación ciudadana y gestión social del hábitat.

Miguel Olivetti Espina, el sociólogo Jorge Castro Falero, el diseñador industrial Guillermo Lago y la trabajadora social Cecilia Rodríguez presentan la experiencia del "Observatorio Sociocultural del Oeste de Montevideo: una plataforma integral universitaria". Se trata de un espacio de formación multidisciplinario, vinculado a la extensión universitaria, a través de los Espacios de Formación Integral de la Universidad de la República de Uruguay. La experiencia muestra, desde diversas perspectivas disciplinares, cómo sobre un mismo territorio se construyen socialmente imaginarios arraigados, vinculados a la cultura del habitar de sus habitantes, al tiempo que impactan sobre el mismo intervenciones urbanísticas y se proyectan desde fuera significados que estigmatizan y excluyen estos barrios periféricos. El conocimiento producido sobre estas imágenes socialmente producidas, juntamente con las organizaciones populares de estos barrios, aporta herramientas para la transformación social del territorio.

Los coordinadores del número, el Dr. arquitecto Esteban de Manuel y la Mg. arquitecta M. ${ }^{a}$ Bernabela Pelli, junto con el Dr. arquitecto José M. ${ }^{a}$ López Medina, en "Cantes de ida y vuelta en la formación sobre la gestión del hábitat. Aprendizajes y confluencias entre el Paraná y el Guadalquivir", presentan una experiencia cooperativa de diseño e implementación de cursos de posgrado en Producción y Gestión Social del Hábitat, desarrollada en Resistencia, Montevideo y Sevilla. Esta experiencia ha permitido compartir y desarrollar conjuntamente herramientas conceptuales y metodológicas para el diseño e implementación de procesos de transformación socio-espacial adecuadas a los distintos contextos, basándonos como premisas en la participación y concertación de los actores involucrados (técnicos, políticos y vecinales), la interdisciplinariedad, la intersectorialidad, la multiescalaridad y la sustentabilidad. Los cursos se vertebran en torno a prácticas de intervención, alimentadas teórica y metodológicamente, siguiendo la secuencia de aproximación y reconocimiento de una necesidad habitacional, formulada como tema-problema sobre el que intervenir, seguida de un diagnóstico integral, participativo y prospectivo y, por último, de la formulación de una estrategia de intervención.

La sección de miscelánea incluye aportes críticos a la Conferencia Hábitat III del Mg. en City Planning Alfredo Rodríguez y la doctora arquitecta Ana Sugranyes. En "La nueva agenda urbana: pensamiento mágico", como participantes en las tres conferencias vinculados a la Habitat International Coalition (HIC), presentan una perspectiva global de las tres conferencias, desde los avances y retrocesos producidos durante estos 40 años en la construcción del derecho a la ciudad como 
categoría de transformación democratizadora del hábitat social. Los autores analizan las vicisitudes por las que ha pasado la redacción de la Nueva Agenda Urbana entre 2014-2016 hasta convertirla en un documento lleno de buenas intenciones que no se sustenta en un análisis crítico del pasado ni en un análisis prospectivo. El resultado para los autores es decepcionante, a raíz del acuerdo de "un amplio abanico de grupos de interés que, junto a los Estados miembros de Naciones Unidas, han propuesto, negociado y aprobado un documento sin prioridades, sin instrumentos claros para su implementación ni un marco preciso que pudiera ser aplicado por los Estados".

Se complementa este artículo con la presentación del Dr. en arquitectura Rafael Llacer Pantion, de la reseña del libro del Dr. ingeniero Julián Salas Serrano De Hábitat II a Hábitat III. Construyendo con recursos escasos en Latinoamérica, en el que sistematiza las experiencias desarrolladas en este continente para la mejora del hábitat desde los conceptos de habitabilidad básica y de tecnologías apropiadas y apropiables. La cuestión del acceso al suelo adecuado, a su urbanización, a estrategias de construcción progresivas, a tecnologías y materiales adecuados y los procesos de mejora de barrios precarios son los temas en los que ha venido trabajando el autor en estos 20 años, combinando investigación, intervención y formación de especialistas universitarios para acompañar estos procesos.

También en esta sección de miscelánea se presentan resultados de siete trabajos de investigación, como parte del aporte teórico que genera la universidad al campo de la actuación urbana.

La Dra. en Filosofía del Derecho Ángeles Galiana Saura, en "La vivienda como objeto de estudio desde el derecho: la vivienda como derecho humano y la cátedra UNESCO sobre vivienda", analiza la difícil construcción jurídica del derecho a la vivienda como un derecho subjetivo fundamental y lo relaciona con la construcción del emergente derecho a la ciudad y con los planteamientos de la sostenibilidad en sus dimensiones política, social, económica y ambiental.

Vuelven a la revista las aportaciones sobre movilidad sostenible con el artículo presentado por el consultor Vicente Hernández Herrador y el Dr. en Física Ricardo Marqués, "El impacto del 'carril-bici' de Sevilla sobre el espacio urbano de la ciudad: un análisis preliminar". El propósito del mismo es demostrar que la implantación de la red de carrilesbici en Sevilla se ha realizado fundamentalmente a partir de reducir el espacio destinado a la circulación motorizada en la calzada, seguido de la pavimentación de espacios no pavimentados y, en menor medida, de recorridos peatonales. Para ello elaboran un método de análisis gráfico que permite obtener los incrementos, positivos y negativos, de espacios a disposición de las redes motorizada y peatonal, como resultado de la creación, prácticamente ex novo, de la red ciclista de Sevilla.

La arquitecta Luisa Alarcón González y el Dr. arquitecto Francisco Montero-Fernández, en "Nuevas interacciones entre universidad, sociedad y territorio a través de una propuesta para una industria educacional avanzada. The Potteries Thinkbelt", analizan el proyecto utópico de Cedric Price, con el que se propuso demostrar cómo la universidad podría cumplir la función catalizadora de la reactivación económica de los territorios industriales en declive. Para ello concibe una relación estrecha entre el conocimiento generado en la universidad y los recursos territoriales, con énfasis en los culturales. Por su parte, la arquitectura se implanta en el territorio a partir de una lectura atenta de sus condiciones, prestando especial atención a la movilidad, y se concibe de for- 
ma flexible, de modo que tenga capacidad de adaptarse a las necesidades de uso que demande un contexto cambiante.

El Dr. en psicología Vicente Manzano-Arrondo, Beatriz Pedrosa Vico y José Luis Soto, en "Sinhogarismo inmigrante. Un caso de investigación-acción universitaria en el contexto de la recogida de aceituna en Úbeda”, presentan una experiencia de investigación-acción participativa interuniversitaria, que se inicia en noviembre de 2015 y continúa actualmente. Plantean la problemática compleja de la inmigración temporera, en la que intervienen inmigrantes y población autóctona, en torno a la recogida de la aceituna en Úbeda, municipio de la provincia de Jaén, en España. La iniciativa universitaria se concreta en unidades de acción comprometidas (UAC), con la finalidad de comprender los aspectos relacionados con la problemática y se plantea objetivos de cambio social, aprendizaje y estrategias de comunicación, en los que participan, desde una perspectiva dialógica, al menos la población afectada y la universidad. Desde su accionar el grupo manifiesta que la universidad debería jugar un papel protagonista, basado en su condición de institución que genera conocimiento relevante. Plantean que esta puede y debe generar el conocimiento que es necesario para: comprender qué ocurre, diseñar y fundamentar medidas, y guiar a los agentes implicados en la implementación de las soluciones.

El Ldo. en Ciencia Política Iván Alejandro Beletzky, en "Grandes proyectos de transformación urbana: sus impactos, beneficios y perjuicios. El caso de la Costanera de Posadas", realiza un análisis de impactos negativos y positivos de lo que significó y significa la transformación de la Costanera de Posadas, capital de la provincia de Misiones, Argentina. La obra implicó, por un lado, una renovación estético-turística y la aparición de una nueva centralidad, y por otro, trajo como consecuencia una ciudad menos compacta con gran dependencia del uso del auto, generó un proceso de periferización y, a la vez, perjuicios insondables para las familias relocalizadas. La finalidad del estudio es poner en debate la cuestión de los grandes proyectos de transformación urbana y sus impactos, negativos y positivos, en las ciudades donde se implementan.

La Mg. en estadística Andrea Andrade Prudente y la arquitecta Manoela de Siqueira Leiro, en "Inserção Urbana no Programa Minha Casa Minha Vida (MCMV): Avaliação do Conjunto Habitacional Coração de Maria no Municipio de Salvador, Bahia, Brasil”, presentan un trabajo de investigación desarrollado en la Universidade Federal da Bahia, Brasil, en el que estudian el impacto urbano que significó el Programa Minha Casa Minha Vida (MCMV) en el municipio de Salvador de Bahía. La metodología de investigación implementada les permitió verificar, mediante un estudio de caso, que aunque los beneficiarios se muestren satisfechos con la obtención de una casa propia, este sentimiento se confunde con una insatisfacción debido a la baja calidad urbanística, la cual no ofrece buenas condiciones de acceso a la ciudad ni oportunidades de desarrollo social y económico por parte de los habitantes. A partir de ello afirman que el problema habitacional, como cuestión social del municipio, no se resuelve únicamente con la construcción de unidades habitacionales.

Las abogadas María Soledad Arenaza y María Laura Canestraro, en "La regulación de suelo como componente del derecho a la ciudad (Argentina, 2004-2015)", ponen en debate la perspectiva de que el Estado no avanzó sustancialmente en modificaciones al derecho de propiedad, constituyéndose este como uno de los principales impedi- 
mentos en la garantía del derecho a la ciudad. Esto se evidenció en las políticas públicas implementadas en Argentina durante el período 2004-2015 que, aún en el marco del incremento de las intervenciones estatales en la problemática habitacional, encontraron sus limitaciones en el acceso al suelo.

Pelli, M. B. y De Manuel Jerez, E. (2017). Universidad y producción social del hábitat: investigación, formación y acción. Hábitat y Sociedad, 10, 5-12

<http://dx.doi.org/10.12795/HabitatySociedad.2017.i10.01> 